\title{
MODIFICAÇÃO QUÍMICA DA FARINHA DE ARROZ COMO ALTERNATIVA PARA O APROVEITAMENTO DOS SUBPRODUTOS DO BENEFICIAMENTO DO ARROZ
}

\author{
ELIZABETH HARUMI NABESHIMA * \\ AHMED ATIA EL-DASH **
}

\begin{abstract}
Enfocou-se nesta revisão de literatura a viabilidade da utilização da farinha de arroz para fins de modificação química. Foram abordados aspectos do aproveitamento dos subprodutos do beneficiamento do arroz, propriedades e usos da farinha de arroz, além do procedimento de preparo e propriedades do amido intercruzado ou acetilado. Conclui-se que a farinha de arroz apresenta propriedades especiais que podem contribuir de forma diferenciada para a produção de novos produtos. A modificação química pode ampliar as possibilidades de uso de subprodutos do beneficiamento do arroz na indústria alimentícia.
\end{abstract}

PALAVRAS-CHAVE: ARROZ-FARINHA; ARROZ-MODIFICAÇÃO QUÍMICA.

\section{INTRODUÇÃO}

Segundo levantamentos da CONAB, a produção brasileira de arroz foi de \pm 10.656 .100 toneladas na safra de $2001 / 02$. Valor pouco abaixo da quantidade consumida, que é de 11.700 .000 ton (FERRAZ, 2003).

Ao contrário do que ocorre com o trigo e o milho (transformados em outros produtos antes do consumo), o arroz é consumido no Brasil principalmente na forma de grãos inteiros, descascados e polidos (CASTRO et al., 1999).

* Doutoranda em Tecnologia de Alimentos, Bolsista CNPq, Faculdade de Engenharia de Alimentos (FEA), Universidade Estadual de Campinas (UNICAMP), Campinas, SP (e-mail: ehnabeshima@hotmail.com).

** Professor titular, Departamento de Tecnologia de Alimentos, FEA, UNICAMP, Campinas, SP (e-mail: ahmed@fea.unicamp.br). 
O desenvolvimento de produtos mais sofisticados usando o arroz como matéria-prima seria incompatível com o poder de compra da maioria da população mundial, tradicionalmente, consumidora de arroz (ADAIR, 1972; CASTRO et al., 1999). Entretanto, é viável o aproveitamento de seu subproduto (ADAIR, 1972), pois o beneficiamento do arroz resulta em aproximadamente $14 \%$ de grãos quebrados (CASTRO et al., 1999). Esses apresentam menor valor comercial, cerca de 1/5 do preço em relação aos grãos inteiros (LOPES, 1989). São usados como ingrediente de ração animal, em vinícolas e cervejarias e em menores proporções para "pet foods" (SHIH et al., 1999). Também têm sido utilizados como ingrediente para produção de cereais matinais, produtos hipoalergênicos, fórmulas infantis e alimentos com baixa caloria (LUNDUBWONG e SEIB, 2000). Uma das alternativas para agregar valor aos grãos quebrados seria a modificação química, transformando essa matéria-prima em ingrediente alimentício com maior interesse industrial e comercial (JULIANO e SAKURAI, 1985).

O objetivo desta revisão foi verificar a viabilidade da utilização dos subprodutos do beneficiamento do arroz para fins de modificação química, agregando-Ihes valor e proporcionando matéria-prima econômica e de propriedades funcionais diferenciadas para a indústria.

\section{BENEFICIAMENTO DO ARROZ}

As principais etapas do beneficiamento do arroz (Figura 1) compreendem o descascamento (no qual se extrai cerca de $20 \%$ de casca), a brunição e o polimento (em que são retirados, parcial ou totalmente, o embrião e a maior parte da película que recobre o grão). Dessa etapa, resulta o farelo que representa aproximadamente $8 \%$ do volume do produto em casca. Após, ocorre à etapa de separação das frações de grãos quebrados (14\%) e inteiros (58\%), bem como a classificação dos grãos quebrados em grandes, médios e quirera (CASTRO et al., 1999).

Considera-se grão quebrado, o pedaço de arroz que ficar retido na peneira de 1,6 milímetros de diâmetro e que apresentar comprimento inferior a três quartas partes do comprimento mínimo da classe a que pertence. A quirera é a parte que vaza na referida peneira (BRASIL, 1989), sendo formada por fragmentos dos grãos que se rompem durante 
o processo de beneficiamento. São decorrentes do efeito da aplicação de força mecânica externa aos grãos, que provoca sua divisão em pequenos pedaços. Podem ser ainda resultantes de grãos fissurados, portadores de pequenas trincas decorrentes de ação mecânica interna originada por condições externas não-mecânicas durante o descasque (LOPES, 1989). Conforme CASTRO et al. (1999), além do manejo dos equipamentos, a quebra do grão no beneficiamento ocorreria por razões inerentes ao próprio grão. Esses podem conter problemas (gessados, mal formados ou danificados), sendo conseqüentemente mais susceptíveis a fragmentação durante o descascamento e o polimento. Outro fator seria a secagem, pois se for mal conduzida também pode contribuir para a quebra.

\section{FIGURA 1 - BENEFICIAMENTO DO ARROZ EM CASCA COM AS PROPORÇÕES APROXIMADAS DE SEUS PRODUTOS ESUBPRODUTOS}

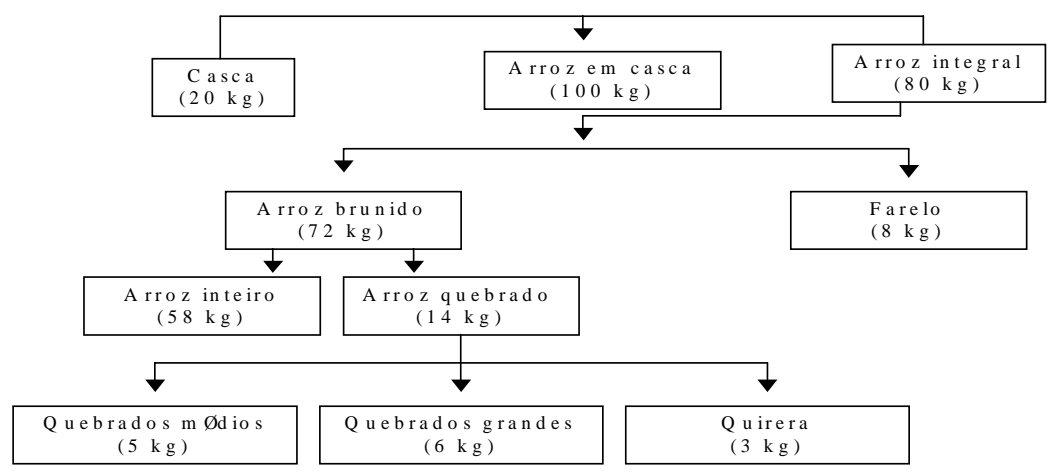

Fonte: CASTRO et al., 1999.

\section{AMIDO E FARINHA DE ARROZ}

O grânulo do amido de arroz é muito pequeno, quando comparado com os de milho $(15 \mu \mathrm{m})$ e de trigo $(30 \mu \mathrm{m})$, variando de 2 a $10 \mu \mathrm{m}$. Sua estrutura apresenta formato dodecahedro pentagonal, o qual pode ser devido à compressão do grânulo de amido durante o desenvolvimento do grão (JULIANO, 1972). A presença de corpos protéicos dispersos 
na matriz amilácea dificulta o isolamento do amido (DEOBALD, 1972). Conforme LUNDUBWONG e SEIB (2000), a separação e purificação dos minúsculos grânulos de amido de arroz também é dificultada pela lenta sedimentação em água.

O amido purificado apresenta custo mais elevado que o da farinha para o mesmo cereal (RANKIN et al., 1964; DEOBALD, 1972). LUNDUBWONG e SEIB (2000), SHIH et al. (1999) estudaram métodos de isolamento do amido de arroz de forma a otimizar o processo. Conforme tais autores, a separação das proteínas do amido de arroz requer tratamento alcalino, detergente ou enzimático (protease), cujo custo inviabiliza o uso do amido de arroz. De acordo com LUNDUBWONG e SEIB (2000), a utilização tanto de álcali como de detergentes aniônicos resulta em amido com alta pureza. No entanto, esse procedimento gera grande quantidade de efluentes. Já o tratamento utilizando protease requer condições de temperatura de $38^{\circ} \mathrm{C}$ e 24 horas para liberar o amido. $\mathrm{O}$ alto tempo de reação torna o processo muito caro, além de intensificar os problemas de contaminação microbiológica.

Conforme DEOBALD (1972), apesar do menor custo, a farinha de arroz não conta com volume de produção expressiva por não apresentar aplicação competitiva em relação ao trigo. Entretanto, suas características especiais deveriam ser melhor exploradas. Por exemplo, não é alergênico, existem variedades com ampla faixa de teor de amilose (o que permite a seleção de acordo com a finalidade), não é tóxico para portadores de doença celíaca (podendo ser utilizado como substituto do trigo na elaboração de produtos sem glúten), o pequeno tamanho dos grânulos de amido apresenta textura extremamente suave com o cozimento e sabor brando (POLANCO et al., 1995), contém baixos níveis de sódio e alta proporção de amidos facilmente digeríveis (TORRES et al., 1999).

As farinhas de arroz vêm sendo utilizadas para atender necessidades especiais da indústria ou do consumidor (alimentos para bebês, produtos cárneos, formulação de panquecas e waffles, etc.). Devido à características peculiares, a farinha de arroz ceroso pode ser útil na produção de molhos congelados. Além disso, é excelente agente de separação de massas de biscoitos refrigerados pela baixa capacidade de absorção de umidade (DEOBALD, 1972). 


\section{FARINHAS E AMIDOS MODIFICADOS}

A substituição do amido pela farinha representa alternativa viável para a expansão da demanda por amidos modificados quimicamente. Além de mais econômica, a farinha apresenta elevados teores de amido. Contudo, a quantidade de farinha utilizada para essa finalidade é pequena em relação ao amido, especialmente, pela sua maior viscosidade de pasta.

A farinha de arroz será tratada em relação ao amido, seu componente principal (Tabela 1).

\section{TABELA 1 - COMPOSIÇÃO DO GRÃO DE ARROZ (em 100 g)}

\begin{tabular}{lcc}
\hline COMPONENTE & INTEGRAL & POLIDO \\
\hline gua (\%) & 12,0 & 12,0 \\
Prote na (\%) & 7,5 & 6,7 \\
Gordura (\%) & 1,9 & 0,4 \\
Carboidrato $(\mathrm{g})$ & 77,4 & 80,4 \\
Fibra (g) & 0,9 & 0,3 \\
Cinza (\%) & 1,2 & 0,5 \\
CAEcio (mg) & 32,0 & 24,0 \\
F sforo (mg) & 221,0 & 94,0 \\
PotAssio (mg) & 214,0 & 92,0 \\
Tiamina (mg) & 0,34 & 0,07 \\
Riboflavina (mg) & 0,05 & 0,03 \\
Niacina (mg) & 4,7 & 1,6 \\
\hline
\end{tabular}

Fonte: CASTRO et al., 1999.

O arroz não constitui fonte primária de amido modificado, já que na maioria das vezes é consumido na forma de grãos inteiros, o que justifica a escassa quantidade de informações disponíveis sobre essa matéria-prima (SHARP e SHARP, 1985). Deve-se considerar a utilização dos subprodutos do arroz como fonte econômica (FONSECA, 1983), e que a indústria de alimentos precisa de produtos amiláceos com diferentes propriedades funcionais (SHARP e SHARP, 1985).

A modificação do amido natural tem se mostrado importante fator no crescimento do mercado de produtos amiláceos devido à capacidade de espessamento, de ligação, formação de gel e textura. $O$ uso de amidos não-modificados é limitado na indústria de alimentos, pois 
intumescem com relativa facilidade e rompem com a mínima injúria. Além disso, produzem estrutura fraca, pasta coesiva ou géis indesejáveis (FOCUS..., 1993).

Para BRADLEY (1992) é necessária a correta especificação do amido para determinada aplicação, visando explorar suas características na produção de alimentos. O amplo uso dos amidos em alimentos tem levado ao desenvolvimento de amidos especiais. As modificações podem ser promovidas por processos físicos, químicos, genéticos e enzimáticos, dependendo de necessidades específicas (LIGHT, 1990). Os amidos modificados são definidos pela fonte, tratamento prévio, proporção de amilopectina e amilose, massa molecular ou grau de polimerização, tipo de substituinte ou derivado, grau de substituição, forma física e componentes associados (ORTHOEFER, 1987).

Os amidos modificados são caracterizados mediante parâmetros, freqüentemente, dependentes do tipo de derivado que está sendo produzido. O grau de substituição (GS), que caracteriza todos os amidos derivados, é a determinação do número médio (expresso em base molar) de grupos hidroxilas de cada unidade D-glucopiranosil que foi derivada (equação 1):

$$
\mathrm{GS}=162 \mathrm{~W} / 100 \mathrm{M}-(\mathrm{M}-1) \mathrm{W}
$$

Na qual:

$\mathrm{W}=$ peso do substituinte (\%), $\mathrm{M}=$ peso molecular do substituinte $\mathrm{.}$

O número máximo possível para o grau de substituição do amido é 3, já que três unidades hidroxilas estão potencialmente disponíveis. Muitos amidos modificados existentes no comércio apresentam GS menor que 0,2 (RUTEMBERG e SOLAREK, 1984).

As modificações mais, amplamente, utilizadas para o melhoramento das propriedades do amido para fins alimentícios são o intercruzamento e a acetilação. Esses proporcionam grande estabilidade e satisfazem necessidades específicas para vários sistemas alimentícios (WHISTLEY e DANIEL, 1990; RUTENBERG e SOLAREK, 1984; MOORTHY, 1985, WURZBURG, 1986; LIGHT, 1990). 


\section{AMIDOS FOSFATADOS DIÉSTER OU INTERCRUZADOS}

Para alimentos, o Food and Drug Administration (1988) permite o uso de amidos modificados com ortofosfato monossódio ou trimetafosfato de sódio e tripolifosfato de sódio (desde de que o fósforo residual não exceda $0,4 \%$ ), trimetafosfato de sódio (fósforo residual máximo de $0,04 \%$ ), e oxicloreto de fósforo (máximo de 0,1\%). Acima de $6 \%$ de ácido fosfórico em combinação com $20 \%$ (no máximo) de uréia pode ser empregado para produzir amidos modificados para a fabricação de papel que entra em contato com certos alimentos (SOLAREK, 1986). Segundo as normas brasileiras, Resoluções n.38 e n.39/76 da CNNPA, é permitido apenas o uso de oxicloreto de fósforo, tripolifosfato e trimetafosfato de sódio (TMS). A limitação e tolerância residual máxima no produto acabado é de no máximo $0,04 \%$, calculada como fósforo (BRASIL, 1977).

Conforme KERR e CLEVELAND, apud WURZBURG (1986), deve-se empregar TMS em meio alcalino para produção de amido diéster. Segundo WURZBURG (1986) e KASEMSUWAN e JANE (1994) muitos dos reagentes de intercruzamento (ou diéster) também produzem derivados monoéster como subprodutos. A proporção de derivados monoéster e diéster pode ser controlada pelo pH da reação. Em pH entre 8-12 há maior probabilidade de ocorrer o intercruzamento, enquanto que em $\mathrm{pH}$ abaixo de 6 a ocorrência maior é de derivado monoéster.

Conforme WURZBURG (1986), as condições de reação usadas para produção de amidos com ligação cruzada variam, amplamente, dependendo de reagentes específicos (bi ou polifuncionais). Muitas das reações são realizadas em suspensões aquosas de amido em temperaturas variando entre ambiente e aproximadamente $50^{\circ} \mathrm{C}$. Freqüentemente, um álcali (tal como o hidróxido de sódio) é usado para promover a reação. As reações são normalmente realizadas sob condições neutras a levemente alcalinas, mas abaixo do nível em que poderá intumescer o amido. Se a reação for conduzida em suspensão aquosa do amido e o nível desejado de ligação cruzada (usualmente mensurada com testes reológicos ou de viscosidade) for atingido, a suspensão será neutralizada e o amido filtrado e lavado para remover os sais. 
Os amidos fosfatados têm sido extremamente utilizados, sendo a ligação cruzada uma das mais importantes modificações químicas (estrutura macromolecular mais rígida no interior do grânulo). Essas ligações reforçam as pontes de hidrogênio naturais, retardam a velocidade de absorção de água do grânulo, intumescem e reduzem a sensibilidade do grânulo intumescido à ruptura (FOCUS..., 1993).

A presença de ligação cruzada aumenta a resistência ao inchamento e à gelatinização do grânulo de amido. As pastas cozidas de amido apresentam maior viscosidade e maior resistência ao efeito do calor ou de $\mathrm{pH}$ baixo. Além disso, as características de elasticidade e coesividade, típicas do amido de mandioca são reduzidas formando pasta cremosa e suave (FOCUS..., 1993).

Quando o amido com ligação cruzada é aquecido em água, as pontes de hidrogênio podem ser enfraquecidas ou destruídas. Contudo, o grânulo irá manter a sua estrutura, sustentada pelas ligações químicas do tipo covalente. Quando o intercruzamento do amido envolve seu tratamento no estado granular, o teor de ligações químicas cruzadas introduzidas será pequeno em relação ao peso do amido e ao número total de unidades de anidroglucose presentes no grânulo (WURZBURG, 1986).

O grau de ligação cruzada no amido é normalmente muito baixo, o que dificulta a quantificação direta mediante métodos químicos. Propriedades físicas como viscosidade e poder de inchamento são geralmente utilizadas para estimar o grau de ligação cruzada (RUTENBERG e SOLAREK, 1984; KASEMSUWAN e JANE, 1994). Esse tipo de modificação é útil em sistemas alimentícios que exigem propriedades de estabilidade ao cozimento, cisalhamento e pH ácido, tal como em molhos para salada, alimentos para bebês, enlatados e tortas (LIGHT, 1990). A aplicação desse tipo de farinha/amido modificado como substituto do glúten de trigo para produção de pães tem sido estudada (WU e SEIB, 1990; CLERICI, 1997).

\section{PROPRIEDADES DO AMIDO DE ARROZ INTERCRUZADO}

$\mathrm{Na}$ literatura não são facilmente encontradas informações sobre intercruzamento de farinha e amido de arroz. É possível que o amido 
de arroz não responda às típicas condições de modificação e/ou as informações são particulares e não estão sendo publicadas (SHARP e SHARP, 1985).

YEH e YEH (1993) obtiveram amido de arroz intercruzado, utilizando $35 \%$ de solução de amido, $0,1 \%$ de oxicloreto de fósforo, $\mathrm{pH}$ alcalino $(10,5), 5 \%$ de sulfato de sódio, temperatura de reação de $35^{\circ} \mathrm{C}$ e tempo de reação variável (15, 30, 60 e 120 minutos). O grau de substituição aumentou com a ampliação do tempo de reação, variando de 2,86 a 3,66 . De acordo com os resultados, a ligação cruzada reforçou a ligação das moléculas de amido e manteve a integridade do grânulo de amido.

CHATAKANONDA, VARAVINIT e CHINACHOTI (2000) intercruzaram o amido de arroz, utilizando trimetafosfato de sódio (300 mg) e tripolifosfato de sódio $(0,18,27$ e $39 \mathrm{~g})$ dissolvido em $300 \mathrm{~mL}$ de água. A reação ocorreu em $\mathrm{pH} 11$ e temperatura de $50^{\circ} \mathrm{C}$, durante 20 minutos. O grau de substituição foi de 0; 9,2; 26,2 e 29,2\%. O intercruzamento alterou significantemente a gelatinização do amido de arroz para temperatura maior, mas não afetou significativamente a entalpia. $\mathrm{O}$ atraso na gelatinização e retrogradação foi evidente devido ao restrito inchamento e reduzida hidratação dos grânulos de arroz.

LUI, RAMSDEN e CORKE (1999) estudaram as propriedades do amido de arroz intercruzado com reagente trimetafosfato de sódio. Verificaram que a modificação do amido de arroz pode ser útil no aumento da viscosidade de pasta (à quente ou frio). Observaram também aumento na força do gel e na estabilidade ao congelamento e descongelamento.

\section{AMIDOS ACETILADOS}

Para a reação de acetilação, a legislação americana (FOOD AND DRUG ADMINISTRATION, 1988) e a brasileira (BRASIL, 1977) permitem o uso de até $10 \%$ de anidrido acético e produto final com o máximo de $2,5 \%$ de grupos acetil.

Os amidos acetilados, além de sua composição normal, contêm grupos acetil ligados à moléculas de amido mediante ligação covalente. A introdução desses grupos substituintes diminui marcadamente a temperatura de gelatinização, enquanto o inchamento e a solubilidade 
aumentam. As propriedades desse tipo de amido variam de acordo com o grau de substituição (RUTENBERG e SOLAREK, 1984). Os altamente acetilados e outros ésteres com GS de 2 a 3 são de interesse devido à solubilidade em solventes orgânicos e termoplasticidade. Os com GS intermediário (entre 0,3 a 1) são tipificados pela solubilidade em água. Os derivados com GS baixo $(0,01$ a 0,2$)$ preservam a estrutura granular após a reação de derivação (JAROWENKO, 1986). A aplicação desse último está baseada nas propriedades de formação de filme, ligação, adesividade, espessamento, estabilização e textura (GRAAF, BROEKROELOFS, JANSSEN, 1998).

A acetilação depende (como toda reação química) de fatores como a concentração do reagente, o tempo de reação, o pH e a presença de catalisadores (WHISTLER e DANIEL, 1990). De acordo com estudo realizado por JAROWENKO (1986), a reação de acetilação ocorre no amido granular e no pré-gelatinizado em meio aquoso com anidrido acético e na presença de hidróxido de sódio diluído (não sendo necessário pré-tratamento de ativação). Aparentemente, a ativação ocorre em "situ" em pH entre 7 e 11, controlado com hidróxido de sódio 3\% e adição de anidrido acético no curso da reação. O pH ótimo parece depender do tipo de amido e da temperatura utilizada (de 8 a 8,4 entre 25 e $30^{\circ} \mathrm{C}$ e 7,0 em temperatura de $38^{\circ} \mathrm{C}$ ).

As condições de reação de acetilação que propiciam maior rendimento foram obtidas com anidrido acético e solução diluída de hidróxido de sódio, adicionados em velocidade moderada, e manutenção do pH na faixa ótima de $8-8,4\left(25^{\circ} \mathrm{C}\right)$ durante o curso da reação. O tempo de reação foi de 30 a 90 min. A reação pode ser interrompida pela acidificação em pH entre 5,5 e 6,5, seguida de centrifugação ou filtração, lavagem e secagem do produto (JAROWENKO, 1986). As aplicações desse tipo de amido modificado estão baseadas, principalmente, nas propriedades de sua estrutura granular, de insolubilidade a frio, de dispersibilidade coloidal durante o cozimento, de formação de filme, de ligação, de adesão, de espessamento, de estabilização e de texturização (JAROWENKO, 1986). Segundo AGBOOLA, AKINGBALA e OGUNTIMEIN (1991), geralmente, o amido acetilado apresenta maior claridade de pasta, estabilidade, maior resistência a retrogradação e estabilidade ao congelamento e descongelamento aumentada. 
De acordo com SHOGREN (1996), a acetilação dos grupos hidroxilas do amido aumenta a hidrofobicidade e conseqüentemente a resistência do amido à água. Pode também reduzir a tendência do amido em formar estruturas fortemente ligadas por pontes de hidrogênio e aumentar a flexibilidade de filmes.

\section{PROPRIEDADES DO AMIDO DE ARROZ ACETILADO}

LUI, RAMSDEN e CORKE (1999) pesquisaram as propriedades físicas do amido de arroz normal e ceroso acetilados. A acetilação foi realizada mediante processo comercial, em pH entre 9 e 10, reagente vinil acetato e ao final da reação $0 \mathrm{pH}$ foi neutralizado para 7,0 . O grau de substituição obtido foi de 0,0157 para o amido ceroso e de 0,0183 para o amido normal. A acetilação aumentou a viscosidade, a dureza dos géis e a solubilidade dos amidos de arroz ceroso e normal, enquanto reduziu a adesividade dos géis.

\section{CONCLUSÃO}

De acordo com o exposto pode-se concluir que existem formas de agregar valor aos grãos quebrados de arroz, utilizando-os em produtos alimentícios mais nobres.

O arroz apresenta propriedades especiais e a modificação química pode ampliar suas possibilidades de uso na indústria de alimentos.

A modificação química da farinha de arroz tem sido pouco explorada, apresentando potencial como alternativa viável para agregar valor aos subprodutos do beneficiamento do arroz.

\section{Abstract}

CHEMICAL MODIFICATION OF RICE FLOUR AS ALTERNATIVE FOR UTILIZATION OF RICE PROCESSING BY-PRODUCTS

In this literature review the viability of rice flour utilization by means of chemical modification is presented. Aspects of rice processing by-products utilization, properties and uses of rice flour, besides the preparation procedure and properties of the cross-linked and acetylated starch was aborded. It was concluded that the 
rice flour presents special properties which can contribute in a different manner to the production of new products. The chemical modification may enlarge the possibilities of rice processing by-products utilization in the food industry.

KEY-WORDS: RICE-FLOUR; RICE-CHEMICAL MODIFICATION.

\section{REFERÊNCIAS}

1 ADAIR, C.R. Production and utilization of rice. In: HOUSTON, D.F. (Ed.). Rice: chemistry and technology. Saint Paul: American Association of Cereal Chemistry (AACC), 1972. p.1-12

2 AGBOOLA, S.O.; AKINGBALA, J.O.; OGUNTIMEIN, G.B. Physicochemical and functional properties of low DS cassava starch acetates and citrates. Starch/ Starke, v.43, n.2, p.62-66, 1991.

3 BRADLEY, G. Natural starch is high technology. Trends of Food Processing, n.5, p.37-41, 1992.

4 BRASIL. Ministério da Saúde. Resoluções n.38 e 39/76. Fixa as características de identidade e qualidade a serem atendidas pelos amidos modificados. In: ABIA. Compêndio da legislação de alimentos. São Paulo, 1977. v.1/A, Seção I, Parte 1.

5 BRASIL. Ministério da Saúde. Portaria $n^{\circ}$ 01, de 04 de janeiro de 1989. Regulamento técnico que aprova o roteiro e os critérios para uniformização da classificação do arroz. Diário Oficial [da] República Federativa do Brasil, Brasília, 1 de fevereiro 1989. Seção 1, pt.1.

6 CASTRO, E.M.; VIEIRA, N.R.A.; RABELO, R.R.; SILVA, S.A. Qualidade de grãos em arroz. Santo Antônio de Goiás: Embrapa Arroz e Feijão, 1999. 30 p.

7 CHATAKANONDA, P.; VARAVINIT, S.; CHINACHOTI, P. Relationship of gelatinization and recrystallization of cross-linked rice to glass transition temperature. Cereal Chemistry, v.77, n.3, p. 315-319, 2000.

8 CLERICI, M.T.P.S. Efeito de modificações fosfatada, intercruzada e ácida durante a gelatinização por extrusão da farinha de arroz e sua influência na produção de pão sem glúten. Campinas, 1997. 252 p. Tese (Doutorado em Tecnologia de Alimentos) - FEA/UNICAMP.

9 DEOBALD, H.J. Rice flours. In: HOUSTON, D.F. (Ed.). Rice: chemistry and technology. Saint Paul: American Association of Cereal Chemistry (AACC), 1972. p. $264-269$.

10 FERRAZ, J.V. Mercado e perspectivas: arroz. In: AGRIANUAL 2003. Anuário estatístico da agricultura brasileira. São Paulo: FNP Consultoria e Comércio, 2003. p.219-228.

11 FDA. Food and Drug Administration. Food starch modified: code of federal regulation. Washington: U.S. Department of Health and Human Services,1988. (Título 21, Sec.12.892). 
12 FOCUS on modified starches. Food Ingredients, n.1/2, p.60-63, 1993.

13 FONSECA, H. Arroz: produção, pré-processamento e transformação agroindustrial. São Paulo: Secretaria de Estado da Indústria, Comércio, Ciência e Tecnologia, 1983. v.2.

14 GRAAF, R.A.; BROEKROELOFS, A.; JANSSEN, L.P.B.M. The acetylation of starch by reactive extrusion. Starch/Staerke, v.50, p.198-205, 1998.

15 JAROWENKO, W. Acetylated starch and miscellaneous organic esters. In: WURZBURG, O. B. (Ed.). Modified starches: properties and uses. Boca Raton: CRC, 1986. p. 55.

16 JULIANO, B.O. The rice caryopsis and its composition. In: HOUSTON, D.F. (Ed.). Rice: chemistry and technology. Saint Paul: American Association of Cereal Chemistry (AACC), 1972. p. 16-27.

17 JULIANO, B.O.; SAKURAI, J. Miscellaneous rice products. In: JULIANO, B.O. (Ed.). Rice: chemistry and technology. Saint Paul: American Association of Cereal Chemistry (AACC), 1985. p.569-618.

18 KASEMSUWAN, T.; JANE, J. Location of amylose in normal starch granules. II. Locations of phosphodiester cross-linking revealed by phosphorus-31 nuclear magnetic resonance. Cereal Chemistry, v.71, n.3, p.282-287, 1994.

19 LIGHT, J.M. Modified food starches: why, what, where and how. Cereal Foods World, v.35, n.11, p.1081-1092, 1990.

20 LOPES, C.C. Estudo do mecanismo de quebra dos grãos de arroz. Campinas, 1989. 128 p. Dissertação (Mestrado em Engenharia de Alimentos) FEAUUNICAMP.

21 LUI, H.; RAMSDEN, L.; CORKE, H. Physical properties of cross-linked and acetylated normal and waxy rice starch. Starch, v.51, n.7, p.249-252, 1999.

22 LUNDUBWONG, N.; SEIB, P.A. Rice isolation by alcaline protease digestion of wet-millet rice flour. Journal of Cereal Science, v.31, p.63-74, 2000.

23 MOORTHY, S.N. Acetylation of cassava starch using perchloric acid catalysis. Starch, v. 37, n.9, p.307-308, 1985.

24 ORTHOEFER, F.T. Corn starch modification and uses. In: WATSON, S.S.; RAMSTAD, P.E. Corn: chemistry and technology. Saint Paul: AACC, 1987. p. 483.

25 POLANCO, I.; MOLINA, M.; PIETRO, G.; CARRACO, S.; LAMA, R. Dieta y enfermidad celíaca. Alimentaria, v.33, n.264, p.91-93, 1995.

26 RANKIN, J.C.; SAMALIK, J.H.; HOLZAPFEL, M.M.; RUSSELL, C.R.; RIST, C.E. Preparation and properties of acid-modified cereal flours. Cereal Chemistry, v.41, n.3, p.386-399, 1964.

27 RUTEMBERG, M.W.; SOLAREK, D. Starch derivatives: production and uses. In: 
WHISTLER, R.L.; BEMILLER, J.M.; PASCHALL, E.P. (Ed.). Starch: chemistry and technology. $2^{\text {nd }}$ ed. New York: Academic Press, 1984. p. 313-314.

28 SHARP, R.N.; SHARP, C.Q. Food applications for modified rice starch. In: JULIANO, B. O. (Ed.). Rice: chemistry and technology. $2^{\text {nd }}$ ed. Saint Paul: American Association of Cereal Chemistry (AACC), 1985. p.405-420.

29 SHIH, F.F.; CHAMPAGNE, E.T.; DAIGLE, K.; ZARINS, Z. Use of enzymes in the processing of protein products from rice bran and rice flour. Nahrung, v. 43, n.1, p.14-18, 1999.

30 SHOGREN, R.L. Preparation, thermal properties, and extrusion of high-amylose starch acetates. Carbohydrate Polymers, v.29, n.1, p.57-62, 1996.

31 SOLAREK, D.B. Phosphorylated starches and miscellaneous inorganic esters. In: WURZBURG, O.B. Modified starches: properties and uses. Boca Raton: CRC, 1986. p. 98-108.

32 TORRES, R.L.; GONZÁLES, R.J.; SÁNCHEZ, H.D.; OSELLA, C.A.; TORRES, M.A.G. Comportamiento de variedades de arroz en la elaboración de pan sin gluten. Archivos Latinoamericanos de Nutrición, v.9, n.2, p. 162-165, 1999.

33 WHISTLER, R.; DANIEL, R. Function of polysaccharides. In: FOOD additives. New York: Marcel Dekker, 1990. p.3399-3406.

34 WU, Y.; SEIB, P.A. Acetylated and hidroxypropylated distarch phosphates from waxy barley: paste properties and freeze-thaw stability. Cereal Chemistry, v.67, n.2, p.202-208, 1990.

35 WURZBURG, O.B. Modified starches: properties and uses. Boca Raton: CRC, 1986.

36 YEH, AN-I; YEH, SU-LAN. Some characteristics of hydroxypropylated and crosslinked rice starch. Cereal Chemistry, v. 70, n.5, p.596-601, 1993. 